\title{
Projeto de um aplicativo mobile para a coleta estruturada de dados e mapeamento de áreas suscetíveis a riscos geológicos
}

\author{
Ana Luiza F. Figueiredo', Thiago J. Inocêncio', Gustavo R. Gonzales ${ }^{1}$, Flávio E. A. \\ Horita $^{1}$ \\ ${ }^{1}$ Centro de Matemática, Computação e Cognição (CMCC) - Universidade Federal do \\ ABC (UFABC) - 09.210-170 -Santo André - SP - Brasil \\ analuffigueiredo@gmail.com, (thiago.inocencio, gustavo.gonzales, \\ flavio.horita\} @ufabc.edu.br
}

Abstract. The mapping of vulnerable areas needs updated data about the geological and structural situation in place. However, the existing processes of data collection, storage, and data analysis are still not effective due to the lack of proper technological resources. This paper presents a software development project of a mobile app to support the establishment of susceptibility maps of geological risks. Preliminary results derived from an interactive research methodology provided evidence on the usefulness of mobile app by domain specialists.

Resumo. O mapeamento de áreas vulneráveis a riscos geológicos necessita de dados atualizados referentes à sua realidade geológico-estrutural. Entretanto, o processo de coleta e análise desses dados ainda é lento e pouco eficaz devido à falta de mecanismos tecnológicos apropriados. Este artigo apresenta o projeto de desenvolvimento de um aplicativo mobile para apoiar a coleta estruturada de dados e elaboração de mapas de suscetibilidade a riscos geológicos. Resultados preliminares em uma metodologia interativa de estudos tornaram evidentes os beneficios do aplicativo para os trabalhos dos especialistas.

\section{Introdução}

Desastres são caracterizados pela disruptura das "capacidades locais" da comunidade, resultando em diversos impactos adversos, tais como a perda de vidas, danos financeiros e estruturais, degradação urbana [Jha, 2010]. Governos e órgãos de emergência então têm atuado na definição de políticas públicas e na condução de intervenções em áreas susceptíveis a estes eventos como forma de minimizar os potenciais danos.

No cenário brasileiro, o Plano Municipal de Redução de Risco (PMRR), em geral, busca apresentar um estudo detalhado sobre os mapas de susceptibilidade das áreas vulneráveis, bem como detalhar planos de contingências e prevenção a potenciais eventos [Alheiros, 2011]. Para isso, órgãos especializados têm adotado a metodologia proposta por [Macedo, 2011] que considera diversas características (por ex., evidências visíveis de movimento, características do local e a categoria da ocupação) para determinar a grau da vulnerabilidade (entre muito baixa - R1; média - R2; alta - R3; e, muito alta - R4) [Alheiros, 2011]. Este grau então define a urgência de intervenção e pode direcionar a liberação de recursos para cada área [Alheiros, 2011]. Entretanto, a metodologia existente apresenta deficiência no que se refere à fase de coleta de dados, principalmente, porque 
estes dados são coletados por especialistas, manualmente, por meio de uma ficha de setor, durante visitas de campo [Macedo, 2011].

Embora diversos aplicativos têm sido desenvolvidos [Santos et al., 2012; Lima et al., 2018], muitos deles destinam-se à emissão de alertas em situações de risco imediato por meio da análise de dados fornecidos por moradores da região afetada. Nesse contexto, este artigo detalha o projeto de um aplicativo mobile, chamado Risk Estimator App (REAPP), para apoiar na coleta estruturada de dados e na elaboração de mapas de susceptibilidades aos riscos geológicos. Este aplicativo está sendo elaborado a partir de um estudo de caso realizado com membros do Laboratório de Gestão de Riscos (LabGRIS) da Universidade Federal do ABC (UFABC), especializado na realização de projetos PMRR em diversos municípios do Estado de São Paulo, Brasil.

\section{Materiais e métodos}

Este trabalho combina os seguintes métodos de pesquisa qualitativa e projeto de software: a) o estudo de caso, método para investigar um fenômeno em seu contexto real a partir de entrevistas semi-estruturadas [Seaman, 1999]; e b) a design science research, um método empregado para a elaboração e validação de artefatos (por ex., sistemas, modelos e diagramas) de tecnologia de informação [Horita et al., 2018]. Esses métodos combinados em ciclos de desenvolvimento permitem um progresso interativo e consistente no projeto.

Quatro ciclos foram realizados até o momento da escrita deste artigo. Em Abril/2018, no Ciclo 1, a equipe do laboratório LabGRIS foi acompanhada em trabalho de campo para que fossem levantadas as necessidades do aplicativo em um contexto real de uso. No Ciclo 2, um conjunto de requisitos foi levantado e validado com os membros do laboratório entre Agosto e Novembro de 2018. Nesse ciclo também foi elaborado um protótipo de interfaces do aplicativo. No Ciclo 3, o protótipo foi avaliado com membros do laboratório entre Janeiro e Fevereiro de 2019. Alguns requisitos foram refinados com base em dados coletados no Ciclo 2. Por fim, no Ciclo 4, novas interfaces foram incluídas no protótipo, sendo elas validadas no laboratório em Março de 2019.

\section{Aplicativo Risk Estimation App (RE-App)}

De maneira geral, o aplicativo mobile busca apoiar a coleta estruturada de dados específicos para mapear as áreas susceptíveis a riscos geológicos. Com base nos dados coletados durante os Ciclos 1 e 2, na Tabela 1 é apresentada a lista detalhada com os principais requisitos do aplicativo mobile.

Tabela 1. Requisitos principais do aplicativo RE-App

\begin{tabular}{|l|c|}
\hline R1 & $\begin{array}{c}\text { O sistema deve permitir ao usuário cadastrar informações sobre as características da } \\
\text { localidade. }\end{array}$ \\
\hline R2 & $\begin{array}{c}\text { O aplicativo deve permitir associar as demarcações feitas no mapa com uma ficha. } \\
\text { Assim, o usuário poderá visualizar melhor a área da respectiva ficha e acessar a ficha } \\
\text { diretamente do mapa. }\end{array}$ \\
\hline
\end{tabular}




\begin{tabular}{|c|c|}
\hline R3 & $\begin{array}{c}\text { O sistema deve permitir ao usuário georreferenciar a ficha manualmente. No entanto, } \\
\text { caso seja possível, o aplicativo deverá fazê-lo de forma automática por meio de } \\
\text { geolocalização. }\end{array}$ \\
\hline R4 & $\begin{array}{c}\text { Para evitar dados incompletos todos os campos de uma ficha deverão ser preenchidos, } \\
\text { bem como a geolocalização do usuário, no caso de não haver dado para um } \\
\text { determinado campo, o usuário poderá selecionar a opção "não se aplica". }\end{array}$ \\
\hline
\end{tabular}

Em seguida, baseado nos requisitos da Tabela 1, na Figura 1 é apresentado o modelo de contexto do aplicativo com suas entidades de interesse e seus relacionamentos.

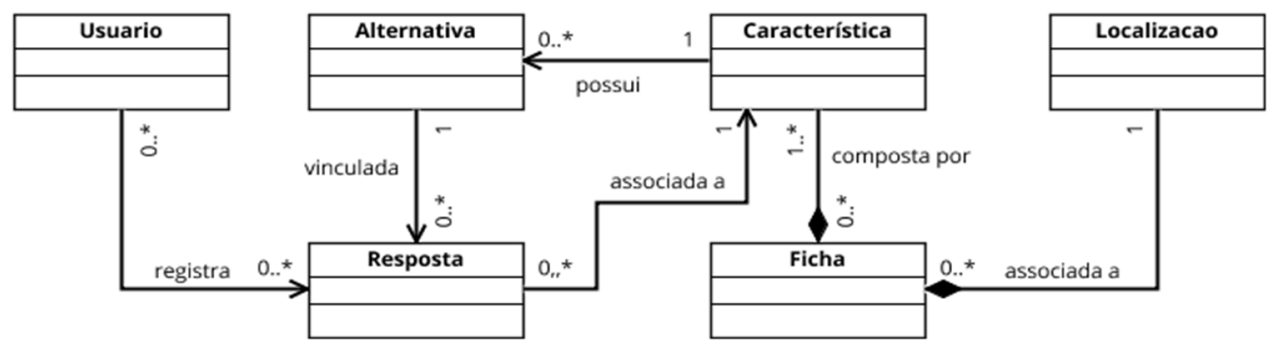

Figura 1. Modelo de Contexto

Sendo a ficha de setor, o elemento central na coleta estruturada de dados para a definição das áreas vulneráveis em projetos de PMRR [Macedo, 2011], na Figura 1, temos que essa ficha é composta por uma ou várias características (por ex., estruturais, demográficas, econômicas e geográficas). Estas características possuem nenhuma ou várias alternativas; por ex., a ocupação (característica) pode ser consolidada ou não consolidada (alternativas). Baseado nisso, os usuários do aplicativo (ou seja, os especialistas), durante a coleta dos dados, fornecem então respostas para cada uma dessas características em relação a uma localidade de interesse. Dessa forma, cada ficha deve estar relacionada a uma localidade de interesse (por ex., uma casa), sendo ela, por sua vez, composta por características mapeadas por nenhuma ou várias fichas.

Na Figura 2 é apresentado o protótipo de interfaces do RE-App. Na interface à esquerda é apresentada a tela inicial do aplicativo com o mapa de uma região e uma delimitação feita pelo usuário. A interface ao centro apresenta a tela de uma ficha com os itens a serem preenchidos, apresentada após a delimitação da localidade de análise. Por fim, a interface à direita detalha as alternativas existentes para uma característica selecionada (no caso, sistema de drenagem superficial).

\section{Conclusões}

Este artigo apresentou um projeto de aplicativo mobile para apoiar na coleta estruturada de dados e elaboração de mapas de susceptibilidade a riscos geológicos, elemento primordial nos PMRR. Um estudo de caso com design science research está sendo conduzido com membros do laboratório LabGRIS. Resultados preliminares obtidos a partir da avaliação preliminar dos diagramas e modelos elaborados tornou evidente os benefícios do aplicativo mobile para os trabalhos dos especialistas do domínio. 

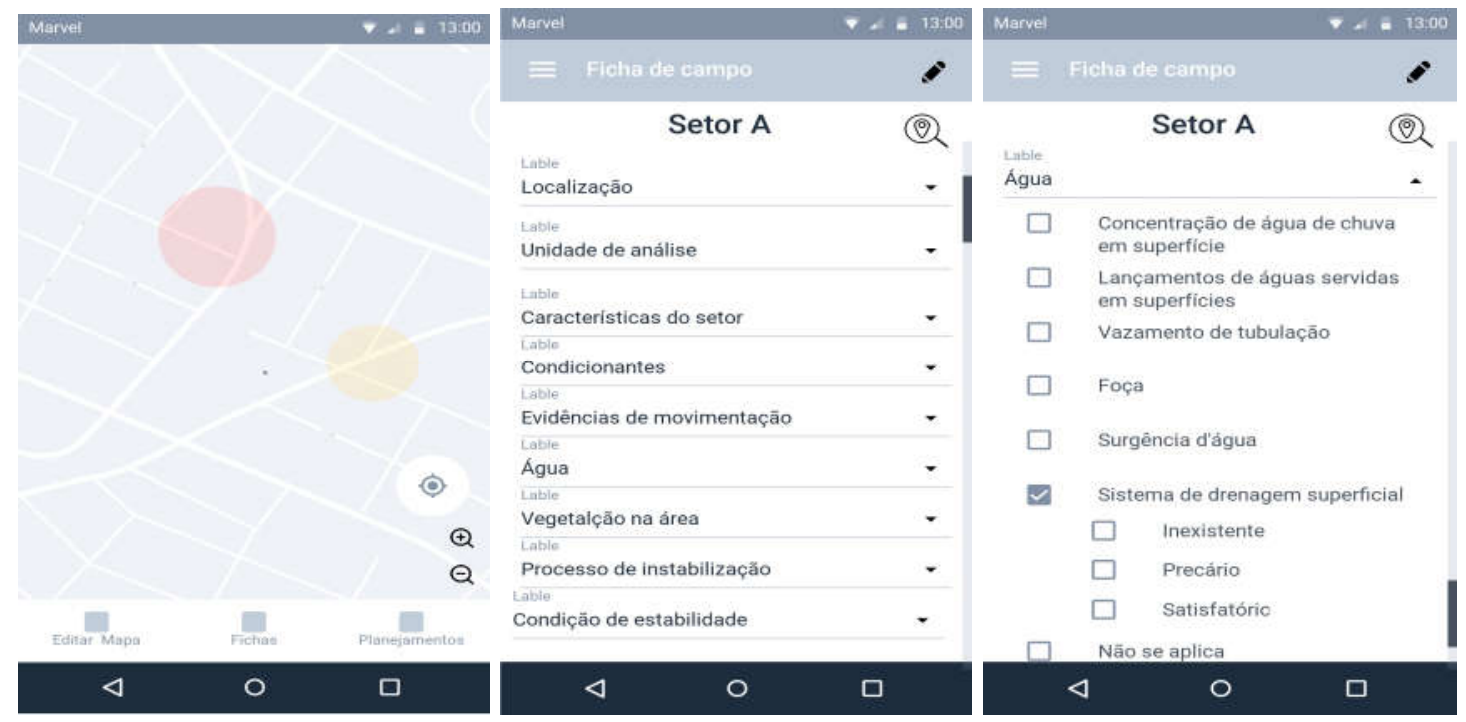

Figura 2. Protótipo de interfaces do RE-APP

De maneira geral, espera-se ainda que o aplicativo possa não apenas incorporar a implementação de modelos para análise de dados feitos por especialistas, mas também ser utilizado para a elaboração dos PMRRs em todo país. Após uma avaliação mais robusta do aplicativo com os usuários, pretende-se realizar novos estudos de casos para refinar o aplicativo como um todo.

\section{Referências}

Alheiros, M. M. (2011). Gestão De Riscos Geológicos No Brasil. Revista Brasileira de Geologia de Engenharia e Ambiental, São Paulo, v.1, n. 1, p.109-122.

Horita, F. E. A., Neto, V. V. G., Santos, R. P. (2018). Design Science Research em Sistemas de Informação e Engenharia de Software: Conceitos, Aplicações e Trabalhos Futuros. In: André Luiz Satoshi Kawamoto; Ana Grasielle Dionísio Corrêa; Valéria Farinazzo Martins. (Org.). I Jornada Latino-Americana de Atualização em Informática, p. 191-210.

Jha, A. K. (2010). Safer homes, stronger communities: a handbook for reconstructing after natural disasters. The World Bank.

Lima, Andressa. S. A. de. et al. Aplicativo colaborativo para alerta de vulnerabilidade a alagamentos e enchentes no Vale do Itajaí. $9^{\circ}$ Workshop de Computação Aplicada à Gestão do Meio Ambiente e Recursos Naturais (WCAMA CSBC 2018).

Macedo, E. S., Canil, K., Silva, F. C., Mirandola, F. A., Gramani, M. F., Ogura, A. T., Corsi, A. C., Santos, L. P. (2011). Methodological procedures to landslide risk mapping in Brazilian slums. Proceedings of the Second World Landslide Forum, Rome, Itália, pp 03-07.

Santos, A. R. dos. et al. (2012). O software SICADSEI 1.0 e suas aplicações como sistema de informação para cadastro das áreas de deslizamento de solo, enchentes e inundação. Espírito Santo do Pinhal, Brasil.

Seaman, C. B., Qualitative Methods In Empirical Studies Of Software Engineering. IEEE Transactions On Software Engineering, Vol. 25, No. 4, July/August 1999, pp. 557572. 